\section{Eddington's Fundamental Equation}

IT is well-known that Eddington has obtained the equation ${ }^{1}$

$$
10 m^{2}-136 m \cdot m^{\prime}+m^{\prime 2}=0 \quad . \quad . \quad .
$$

where $m^{\prime}$ is the mass of a 'scalar particle', that is, a particle the expectation values of the spin and charge of which are zero, and $m$ the mass of a charged particle with spin $\hbar / 2$. The two values of $m$ are shown to be associated respectively with the two signs of the charge, and writing them as $m_{p}$ and $m_{e}$, we have

$$
m_{p}: m^{\prime}: m_{e}:: 1847 \cdot 6: 135 \cdot 9: 1 .
$$

Now the only property of the scalar particle that Eddington has used is that it may be described by a scalar strain vector, for which all the coefficients except $s_{16}$ (the probability density) are zero. He saw that such a strain vector could be used to describe our knowledge of a particle when no observation of its charge or spin had been made, and so wrote that the scalar particle is "a particle which (so far as our information goes) has equal probability of being a proton or electron and equal probability of either direction of spin . . a mathematical fiction, having no counterpart in experimental physics" ". However, it is not necessary to regard the scalar particle in this way, for it may be treated as a particle with as much independent existence as is usually ascribed to the neutrino. Then, according to Eddington's theory, it must have zero charge and zero spin (so that it may be represented by a scalar strain vector) and a mass $m^{\prime}=135.9 m_{e}$ in order to satisfy (1). If this particle were to combine with an electron or positron with the emission of a neutrino, it would yield a heavy negative or positive electron obeying Bose statistics and with mass between 136 and 137 times that of an ordinary electron.

This result is so closely in agreement with the $U$-particle theory of Yukawa ${ }^{3}$, Bhabha ${ }^{4}$, and others, which is in turn supported by facts, so far as present experimental accuracy goes, that it seems very unlikely that equation (1) can be fundamentally wrong, even although the way in which it is obtained is difficult to understand. That the equation should give, in addition to the mass ratio of the proton and electron, the right order for the mass ratio of the electron and a neutral spinless particle (and this before the heavy electron theory was put forward) suggests that Eddington's theory merits more attention than is usually given to it.

\section{H. C. Corben.}

\section{Trinity College, \\ Cambridge.}

1 "Relativity Theory of Protons and Electrons" (Camb. Univ. Press, 1936.) Equation (12.47).

2 "Relativity Theory of Protons and Electrons", p. 89

s Yukawa, Proc. Phy8.-Math. Soc. Jap., 17, 48 (1935).

- Bhabha, Nature, 141, 117 (1938).

\section{X-Ray Study of Thymonucleic Acid}

A FEW years ago an occasion presented itself of examining by $\mathrm{X}$-rays a small specimen of sodium thymonucleate kindly provided by Prof. W. J. Schmidt ${ }^{1}$ of Giessen, and an imperfect fibre photo. graph was obtained with a strong period along the fibre axis of about $3 \frac{1}{2} \mathrm{~A}$. ; but the observation was not followed up at the time. We have now continued the investigation with material prepared in the Department of Chemistry of the Caroline Institute, Stockholm, by the method of Hammarsten and Bang ${ }^{2}$. The viscosity and double refraction of flow of this material in aqueous solution were the subject of a recent communication in NATURE by Signer, Caspersson and Hammarsten ${ }^{3}$.

Films of sodium thymonucleate stretched some 250 per cent have been found to give a striking, though still rather obscure, X-ray fibre photograph in which by far the most prominent reflection corresponds to a spacing along the fibre axis of $3 \cdot 3_{4}$ A., which is almost identical with that of a fully extended polypeptide chain system, such as $\beta$-keratin ${ }^{4}$ or $\beta$-myosin. The true period along the fibre axis is much greater than this--perhaps seventeen times as great, to judge by the present photographs-and there are also side spacings up to about $26 \mathrm{~A}$., the best defined being one of approximately $16 \cdot 2 \mathrm{~A}$.

In view of the hydrodynamic and optical properties of the solutions ${ }^{3}$ and of the optical properties of the solid fibres ${ }^{1}$, the natural conclusion from the X-ray data is that the spacing of $3 \cdot 3_{4} \mathrm{~A}$. along the fibre axis corresponds to that of a close succession of flat or flattish nucleotides standing out perpendicularly to the long axis of the molecule to form a relatively rigid structure, strongly optically negative, and showing double refraction of flow. At first sight the distance $3 \cdot 3_{4} \mathrm{~A}$. between successive nucleotides might seem improbably small, but it is confirmed by the high density, which is $1 \cdot 62-1 \cdot 63 \mathrm{gm}$./c.c. for the dry substance. (Compare the density of ascorbic acid, 1.74, the flat molecules of which are piled together at an even smaller distance $\left.(3 \cdot 16 \mathrm{~A} .)^{5}\right)$. The average weight of a sodium nucleotide is roughly 330 , whence if $A$ be the average area :

$$
3.34 \times A \times 1.62=1.65 \times 330 \text { (approx.) } ;
$$

which means that the average area per nucleotide should be about $100 \mathrm{~A} .^{2}$, as it clearly must be from known interatomic and intermolecular distances (a plausible estimate for the purine nucleotides gives something like $15 \times 7 \frac{1}{2} \mathrm{~A}^{2}{ }^{2}$. Again, the X-ray data lead to an estimate of the molecular weight (between 500,000 and $1,000,000$ ) similar to that already arrived at by other methods ${ }^{3}$; for we may assume an effective average width of the molecule in solution of about $20 \mathrm{~A}$., and therefore from the hydrodynamic data, which indicate a length to width ratio of roughly $300: 1$, infer a length of the order of $6,000 \mathrm{~A}$. Such a length would contain some 2,000 nucleotides of thickness $3 \cdot 3_{4}$ A., and so would agree well with the weight just quoted.

The significance of these findings for chromosome structure and behaviour will be obvious. It seems difficult to believe that it is no more than a coincidence that thymonucleic acid consists of a long succession of nucleotides spaced at a distance so nearly equal to that of the long succession of amino-acid residues in a fully extended polypeptide. Rather is it a stimulating thought that probably the interplay of proteins and nucleic acids in the chromosomes is largely based on this very fact, and that some critical stage in mitosis, involving elongation of the protein chains, is realized in close co-operation with the dominating period of the interacting nucleotides.

We should like to express our indebtedness to Prof. Hammarsten and Dr. Caspersson of the Caroline 